\title{
ORIGINAL ARTICLE \\ In vitro catabolism of quercetin by human fecal bacteria and the antioxidant capacity of its catabolites
}

\author{
Xichun Peng ${ }^{1,2 *}$, Zhichao Zhang ${ }^{2}$, Ning Zhang², Liu Liu², Shaoting Li ${ }^{2}$ and Hua Wei ${ }^{1}$ \\ 'State Key Laboratory of Food Science and Technology, Nanchang University, Nanchang, China; ${ }^{2}$ Department of Food \\ Science and Engineering, Jinan University, Guangzhou, China
}

\section{Abstract}

Background: Part of quercetin flows into the colon after escaping the absorption of the small intestine and will be degraded by colonic microbiota. The catabolites in the colon partially determine the physiological activity of quercetin.

Methods: Seven gut bacteria isolated from human feces were utilized to in vitro ferment quercetin. Their catabolites were analyzed with high-performance liquid chromatography and mass spectrometry, and the antioxidant activities of their fermented broths were compared with that of quercetin.

Results: One metabolite, 3,4-dihydroxyphenylacetic acid, was produced by both $C$. perfringens and B. fragilis transforming quercetin. No other metabolites were detected in the other fermented broths. The antioxidant activities of all strains fermenting quercetin reached the highest values at the concentration of $1 \mathrm{mg} / \mathrm{mL}$ quercetin in broth; the fermented products of $C$. perfringens and B. fragilis presented stronger activities than those of other strains at most concentrations of quercetin in broth. Additionally, all of the fermented broths presented a decline of the antioxidant activities compared to quercetin. Therefore, the antioxidant activity of quercetin will be lost when it reaches the human colon because of the gut bacterial fermentation.

Conclusions: This is the first study to report that quercetin can be degraded by $C$. perfringens and $B$. fragilis and transformed to the same metabolite, 3,4-dihydroxyphenylacetic acid, and that antioxidant activities decline when quercetin is fermented by seven gut bacteria.

Keywords: quercetin; gut bacteria; catabolites; antioxidant activity

Received: 21 November 2013; Revised: I4 January 2014; Accepted: 13 March 20।4; Published: I5 April 2014

$\mathrm{P}$ lant phenolic and polyphenolic compounds have the same antioxidant activity since their structures contain many phenolic hydroxyl groups. The flavonoids are phenolic compounds and therefore prone to oxidation to quinones, which makes flavonoids capable of protecting unsaturated fatty acids in membranes as well as ascorbate against oxidation. Hence, certain brackets of their physiological oxidation-reduction potentials can be estimated (1).

Quercetin is also a flavonoid and often present as its glycoside, quercetin-3-O-rutinoside (rutin), in the human diet. After being ingested, rutin undergoes hydrolysis by the microflora in the colon before absorption (2). The microflora in the colon has enormous catalytic and hydrolytic potential. They will first cut down the glycogen of rutin to utilize as their carbon source (3) and can further catalyze the breakdown of the C6-C3-C6 flavonoid skeleton to a variety of phenolic acid catabolites $(2,4,5)$. Thus, the bioactivity of polyphenol is very much dependent on its catabolism and catabolites that occur before absorption and disposition in target tissues and cells.

The antioxidant activity of quercetin or rutin and its metabolites has also been reported in many literatures $(3,6-8)$. Serra et al. (9) and Jaganath et al. (3) deduced the metabolites and metabolic pathways of the colonic metabolism of quercetin in vitro fermentation model using rat colonic microflora. But it is different between human and rat colonic microflora; besides, the catabolites of quercetin cannot be totally separated since the techniques are limited. Yang et al. (10) analyzed the metabolites of hyperoside (quercetin 3-galactoside) by human fecal flora in vitro. No literature was concerned about the catabolites of special gut bacteria fermenting quercetin (not its glucoside) and the changes of antioxidant activity induced by the fermenting process.

Hence, the objective of this study is to analyze the catabolites of seven human fecal bacteria degrading quercetin and to compare their antioxidant activities with quercetin. 


\section{Materials and methods}

\section{Bacteria and media}

Seven strains - Escherichia coli, Streptococcus lutetiensis, Enterococcus gilvus, Clostridium perfringens, Bacteroides fragilis, Lactobacillus acidophilus, and Weissella confusewere identified and isolated from human feces in our laboratory (11). Two kinds of media, MRS and Brain Heart Infusion (BHI) broths, were purchased from Qingdao Hope Bio-technology Co. and utilized to culture these strains. MRS broth for L. acidophilus and $W$. confuse; BHI for E. coli, E. gilvus, S. lutetiensis, $C$. perfringens, and B. fragilis.

In vitro fermentation

Quercetin (Qiyun Biological Science \& Technology Limited Co., China) was first dissolved with DMSO, and then $50 \mathrm{mg} / \mathrm{mL}$ stock solution was prepared after filtration with a $0.22-\mu \mathrm{m}$ nylon microporous membrane. The solution was stored at $4{ }^{\circ} \mathrm{C}$ before use. Before those strains were individually inoculated into their broth media, the quercetin solution was supplemented to a final concentration of $0.2 \mathrm{mg} / \mathrm{mL}$ in the broths. After inoculation, the $\mathrm{BHI}$ and MRS broths were cultured at $37^{\circ} \mathrm{C}$ in anaerobic conditions for $24 \mathrm{~h}$.

\section{Collection of metabolites}

The above fermented solutions were individually centrifuged at 5,000 rpm for $20 \mathrm{~min}$, and the supernatant solutions were further extracted with an equal volume of ethanol. The extracted solutions were then distilled under reduced pressure at $55^{\circ} \mathrm{C}$ to remove ethanol and were condensed to five-fold. The remains were filtrated with a $0.45-\mu \mathrm{m}$ microporous membrane, and the mixture solution of catabolites (MSCs) were individually collected and stored at $-20^{\circ} \mathrm{C}$ before being used.

\section{HPLC and MS analysis}

After MSCs were thawed in a $37^{\circ} \mathrm{C}$ water bath, $20 \mu \mathrm{L}$ of sample was pipetted by automatic sampler and analyzed with high-performance liquid chromatography (HPLC). The compounds were separated on an analytical Welch Ultimate $\mathrm{XB}-\mathrm{C} 18$ column $(4.6 \times 250 \mathrm{~mm}$ i.d., $5 \mu \mathrm{m}$; Welch, Shanghai, China) using a binary gradient delivered by a LC-20AT low-pressure gradient HPLC pump (SHIMADZU, Japan) with acetonitrile as solvent $\mathrm{A}$ and $0.1 \% \mathrm{v} / \mathrm{v}$ formic acid water solution as solvent $\mathrm{B}$ for the detection of quercetin and its catabolites: $0.01 \mathrm{~min}, 5 \% \mathrm{~A}$; $10 \mathrm{~min}, 10 \% \mathrm{~A} ; 50 \mathrm{~min}, 50 \% \mathrm{~A} ; 65 \mathrm{~min}, 90 \% \mathrm{~A} ; 70 \mathrm{~min}$, $90 \% \mathrm{~A} ; 73 \mathrm{~min}, 5 \% \mathrm{~A}$. The flow rate was $0.6 \mathrm{~mL} / \mathrm{min}$. Data acquisition was carried out with the software ClassicVP Chromatography Workstation (SHIMADZU, Japan). A diode array detection SPD-M20A plus was applied over the wavelength range of 220-600 nm for peak detection. Compounds were identified by comparison of the UV spectra and the retention times. The following wavelengths were monitored for quantitative analysis: $360 \mathrm{~nm}$ for quercetin, $280 \mathrm{~nm}$ for metabolites $(12,13)$.

The fermentation products separated by the HPLC were used for the further experiment. Those components at new peaks were collected and analyzed by mass spectrometry (MS) (Waters, Milford, MA, USA), and the chromatographic scans of selected multiple reaction monitoring ions (MRM) were used for further identification.

\section{Comparison of free radical scavenging activities}

Initially, the broths of each bacterium with 10, 20, 30, 40, and $50 \mathrm{mg} / \mathrm{mL}$ quercetin were individually fermented for $24 \mathrm{~h}$. BHI and MRS broths with quercetins but without incubating bacteria were used as controls. Then MSCs were prepared following the procedure described above.

The DPPH radical scavenging activities of all the samples of the fermentations were evaluated by the method of Blois (14) with minor modifications. Twenty microliter of each MSC was added into $10 \mathrm{~mL}$ colorimetric tubes, made up with $2 \mathrm{~mL}$ of distilled water, and then mixed with $2 \mathrm{~mL}$ of $0.2 \mathrm{mM}$ DPPH dissolved in methanol. The reaction mixture was incubated for $30 \mathrm{~min}$ at $28^{\circ} \mathrm{C}$ in the dark. The blank sample contained all reagents without MSC. The DPPH radical scavenging activity was determined by measuring the absorbance at $517 \mathrm{~nm}$ using a spectrophotometer. The DPPH free radical scavenging activity (\%) was calculated using the following formula (1). The test was paralleled three times.

Percentage inhibition $=\left[\left(\mathrm{A}_{0}-\mathrm{A}_{1}\right) / \mathrm{A}_{0}\right] \times 100$

where $A_{0}$ is the absorbance of the control and $A_{1}$ is the absorbance in the presence of MSCs.

Hydroxyl radical, generated from the $\mathrm{Fe}^{3+}$-ascorbate$\mathrm{H}_{2} \mathrm{O}_{2}$ (Fenton reaction), was evaluated by degradation of deoxyribose that produced thiobarbituric acid reactive species (TBARS) (15). The reaction mixture contained $25 \mathrm{mmol} / \mathrm{L}$ deoxyribose, $10 \mathrm{mmol} / \mathrm{L}$ ferric chloride, 100 $\mathrm{mmol} / \mathrm{L}$ ascorbic acid, and $2.8 \mathrm{mmol} / \mathrm{L} \mathrm{H}_{2} \mathrm{O}_{2}$ in $10 \mathrm{mmol} /$ $\mathrm{L} \mathrm{KH}_{2} \mathrm{PO}_{4}$ (pH 7.4), and it was then mixed with of $0.4 \mathrm{~mL}$ water solutions, including $20 \mu \mathrm{L}$ of each MSC. The blank sample contained all reagents without MSC. The reaction mixture was incubated at $37^{\circ} \mathrm{C}$ for $1 \mathrm{~h}$. Subsequently, $1 \mathrm{~mL}$ of $1 \%$ thiobarbituric acid and $1 \mathrm{~mL}$ of $3 \%$ trichloroacetic acid were added, and the mixture was heated at $100^{\circ} \mathrm{C}$ for $20 \mathrm{~min}$. TBARS was measured spectrophotometrically by taking absorbance at $532 \mathrm{~nm}$. The results were expressed as percentage inhibition of deoxyribose oxidation, as determined by the following formula (2). The test was paralleled three times.

Percentage inhibition $=[(\mathrm{A}-\mathrm{B}) / \mathrm{A}] \times 100$

where $\mathrm{A}$ is the malondialdehyde produced by Fenton reaction treated alone, and $\mathrm{B}$ is the malondialdehyde produced in the presence of MSCs. 


\section{Statistical analyses}

Each sample was analyzed in triplicate, and data are presented as mean values \pm standard error $(n=3)$. Multivariate correlation analysis of appropriate data was performed according to Pearson's correlation.

\section{Results}

\section{Metabolites of various bacteria fermenting quercetin by} HPLC and MS analysis

It is difficult to separate all the metabolites of bacteria by HPLC because of numerous substances in fermented broths. In this experiment, the metabolites of seven strains transforming quercetin were separated with HPLC. Only one compound was discovered, and it presented among the metabolites of both $C$. perfringens and B. fragilis at the retention time of $25.024 \mathrm{~min}$ in the charts (Fig. 1A and B). The retention time of quercetin was about $49.013 \mathrm{~min}$. Its peak appeared in the fermented solution of E. gilvus,
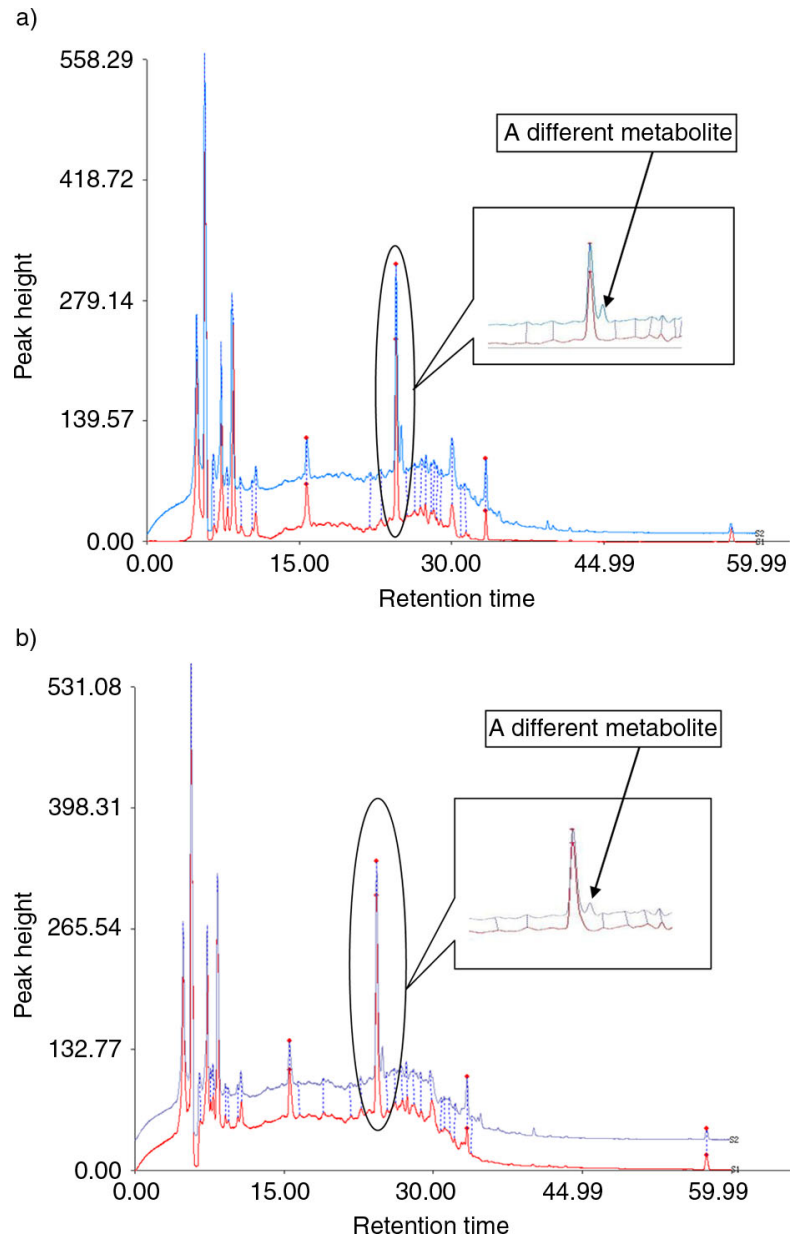

Fig. 1. (a) Catabolites of $C$. perfringens fermenting quercetin, with HPLC analysis. S1 and S2 individually refer to the control and sample. (b) Catabolites of B. fragilis fermenting quercetin, with HPLC analysis. S1 and S2 individually refer to the control and sample.
E. coli, S. lutetiensis, W. confuse, and L. acidophilus, but not of $C$. perfringens and $B$. fragilis.

The component at the retention time of $25.024 \mathrm{~min}$ was collected for MS. Two significant molecular ion peaks of 167 and $123 \mathrm{~m} / \mathrm{z}$ were observed (Fig. 2) and deduced as 3,4-dihydroxyphenylacetic acid, one main metabolite of gut bacterial fermenting quercetin, according to its possible fractures (16).

\section{DPPH free radical scavenging activities of quercetin and its MSCs}

The quercetin in broths removed DPPH free radicals depending on its concentration (Fig. 3). About 70\% DPPH was removed by $1 \mathrm{mg} / \mathrm{mL}$ quercetin. Compared to quercetin, MSCs of seven bacterial species scavenged less DPPH free radicals (Fig. 3). Among MSCs of all strains, that of $L$. acidophilus can remove the least amount of DPPH free radicals in a series of doses of quercetin added in broths. The MSCs of $W$. confuse and E. gilvus, scavenged more DPPH free radicals than that of L. acidophilus, but less than the others when $0.4 \mathrm{mg} / \mathrm{mL}$ quercetin or more was added in broths. The MSCs of the other two strains, that is, $W$. confuse and E. gilvus, scavenged more DPPH free radicals than that of $L$. acidophilus but less than the others when more than $0.4 \mathrm{mg} / \mathrm{mL}$ quercetin was added in broths. Their removal rates of DPPH by MSCs reached the highest value when the concentration of quercetin was $1 \mathrm{mg} / \mathrm{mL}$, the highest concentration of quercetin in broth. The abilities of scavenging free radicals were improved along with more quercetin added in broths.

\section{$\mathrm{OH}$ free radical scavenging activities of quercetin and its MSCs}

The hydroxyl group free radical scavenging activity of quercetin was also related to its concentration (Fig. 4). The removal rate of $\mathrm{OH}$ free radicals reached almost $100 \%$ when the concentration of quercetin was $1 \mathrm{mg} / \mathrm{mL}$. The MSCs of B. fragilis presented the strongest activity in scavenging $\mathrm{OH}$ free radicals in all concentrations of quercetin added in broths. When $1 \mathrm{mg} / \mathrm{mL}$ quercetin was added in both, the MSCs of $B$. fragilis almost removed the same amount of $\mathrm{OH}$ free radicals with quercetin $(100 \%)$ and $80 \%$ in the case of $C$. perfringens. The $\mathrm{OH}$ free radicals scavenging activities of MSCs from $S$. lutetiensis and $B$. gilvus presented the weakest with only about 30\% DPPH removed when more than $0.6 \mathrm{mg} / \mathrm{mL}$ quercetin was added in broth. The activities of all strains increased along with more quercetin being added.

\section{Discussion}

This study attempted to analyze the metabolites of seven gut bacterial strains fermenting quercetin. 3,4Dihydroxyphenylacetic acid, a metabolite that was produced by both $C$. perfringens and $B$. fragilis transforming 


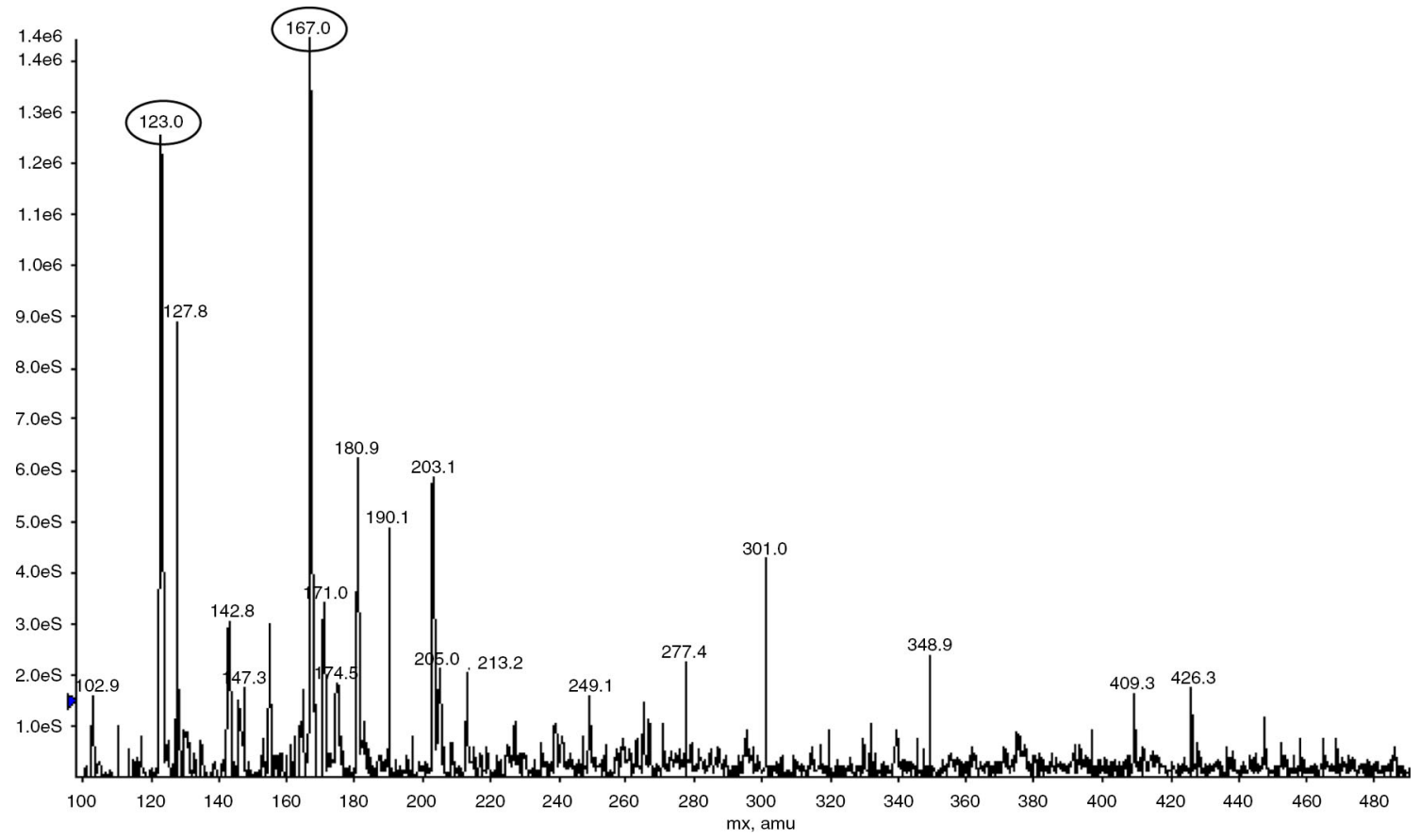

Fig. 2. Mass spectrum of the main compound.

quercetin, was separated and identified with HPLC and MS. No other metabolites were detected in all fermented broths of seven strains. Since the degradation of quercetin was detected in all seven strains (11), the antioxidant activities of seven fermented broths were then compared. Their fermented broths presented different antioxidant activities when their ability of scavenging DPPH and $\mathrm{OH}$ free radicals was tested. The results showed that their activities were almost dependent on the concentration of quercetin and reached the highest value when $1 \mathrm{mg} / \mathrm{mL}$ was added. Furthermore, the samples of $C$. perfringens and $B$. fragilis presented stronger ability in scavenging both DPPH and $\mathrm{OH}$ free radicals.
Serra et al. (9) used rat colonic microflora to in vitro ferment quercetin and other flavonoids. They detected the metabolites of quercetin including 3-(3,4-dihydroxyphenyl) propionic acid, 3,4-dihydroxyphenylacetic acid, 3-hydroxyphenylacetic acid, and phenylacetic acid and deduced the metabolic pathway of quercetin in rat gut (Fig. 5). Only 3,4-dihydroxyphenylacetic acid was detected in the fermented broths of $C$. perfringens and B. fragilis, and no metabolites were found in the other broths, which indicated that only $C$. perfringens and $B$. fragilis from the human gut could transform quercetin to 3,4-dihydroxyphenylacetic acid, and the other five bacteria from the human gut could degrade
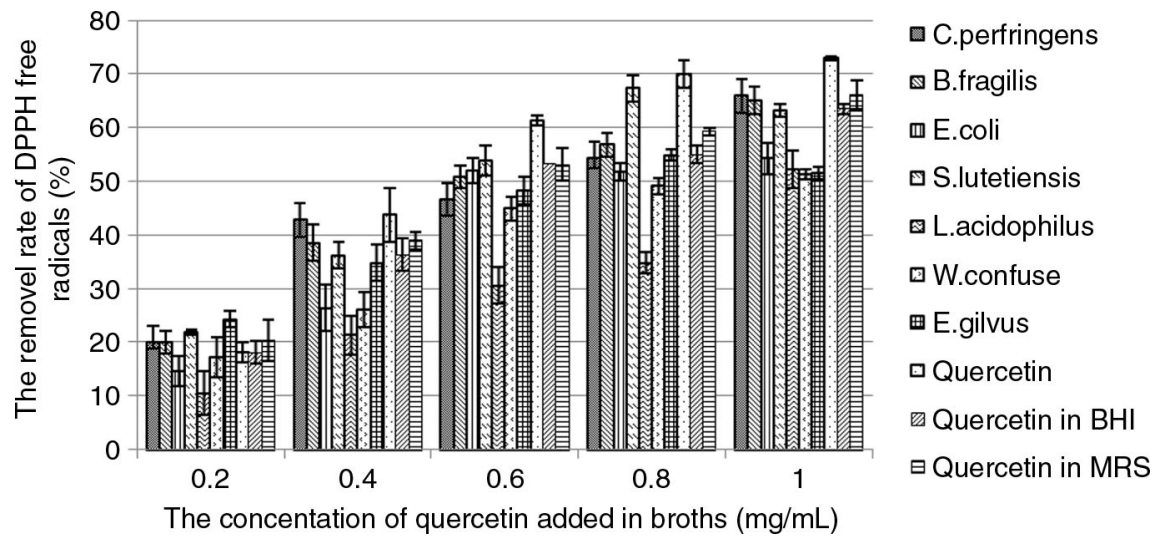

Fig. 3. The removal rate of DPPH free radicals by quercetin and its MSCs from seven bacterial species in broths. The quercetin in BHI and MRS individually refers to the removal rate of DPPH free radicals by BHI and MRS media supplemented with quercetin and incubated for $24 \mathrm{~h}$ but without being inoculated with the bacteria. 


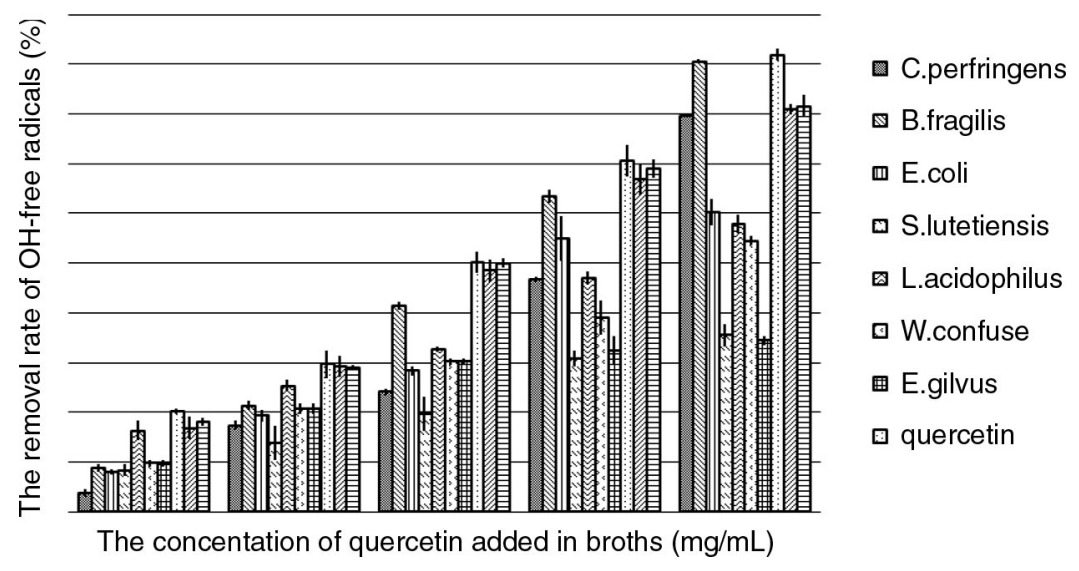

Fig. 4. The removal rate of $\mathrm{OH}$ free radicals by quercetin and its MSCs from seven bacterial species. The quercetin in BHI and MRS individually refers to the removal rate of $\mathrm{OH}$ free radicals by BHI and MRS media supplemented with quercetin and incubated for $24 \mathrm{~h}$ but without being inoculated with the bacteria.

part of quercetin (11) but without 3,4-dihydroxyphenylacetic acid or other metabolites detected. Walle et al. (16) reported that as much as $23.0-81.1 \%$ of the quercetin dose was recovered as $\mathrm{CO}_{2}$ in the expired air from volunteers. Hence, part of quercetin might be directly degraded into $\mathrm{CO}_{2}$ by the other five bacteria, which might be the reason that no metabolites were detected in the other five broths. Nonetheless, the results indicated that different bacteria can transform quercetin into the same metabolite(s), and quercetin can be metabolized by different bacteria to different metabolites.

The phenomenon that metabolic conversion alters the antioxidant activity of quercetin had been reported in many literatures $(3,8,17-20)$. However, most of these literatures focused on evaluating the antioxidant activities

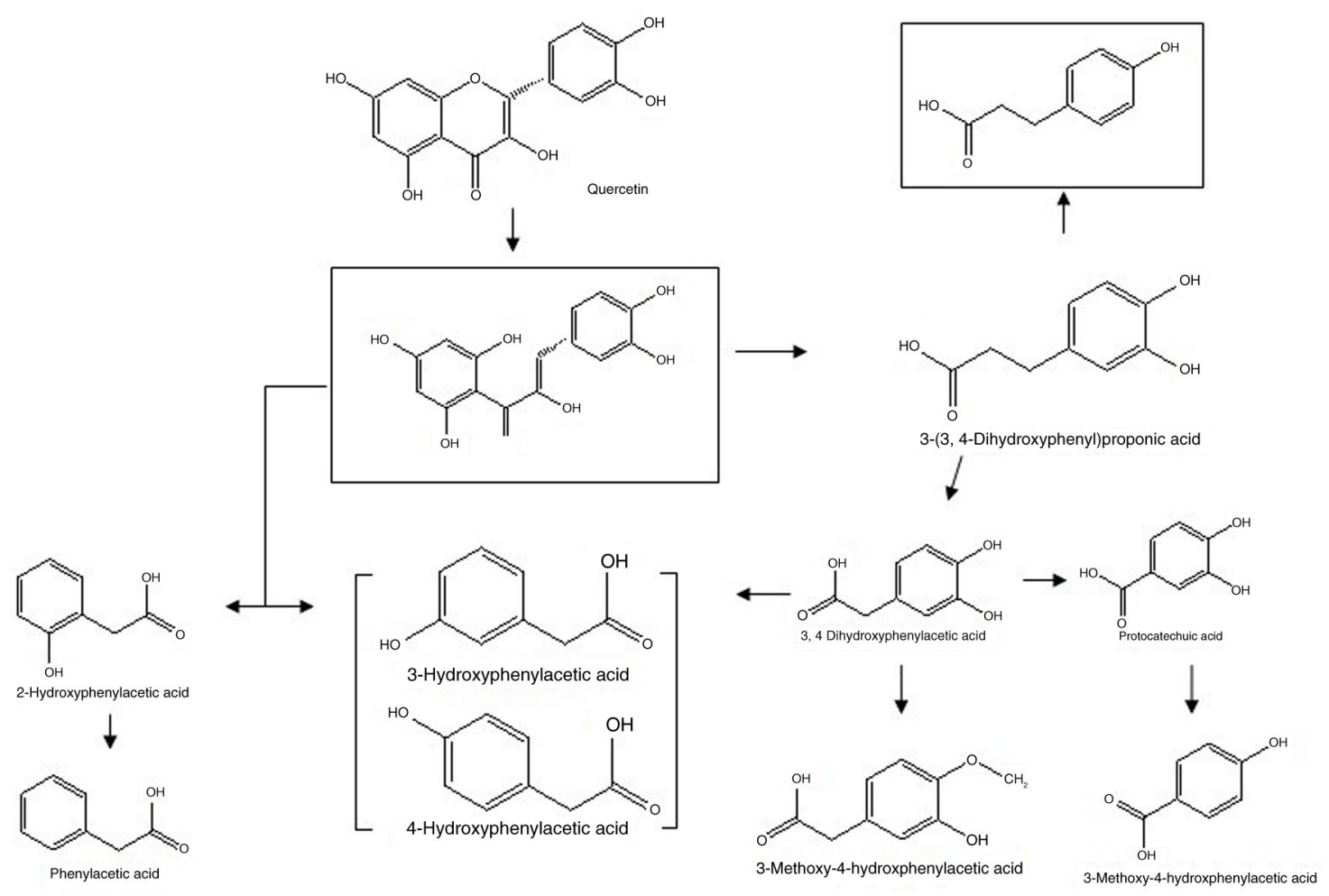

Fig. 5. Proposed metabolic pathway of quercetin by rat colonic microflora. The totally squared metabolites are not detected. From Serra et al. (9). 
of quercetin and its conjugates such as 3'-O-methyl quercetin and 4'- $O$-methyl quercetin $(18,20)$, quercetin3-O-glucuronide, and quercetin-3-O-sulfate $(8,19)$, quercetin 3-O- $\beta$-D-glucuronide (17). The conjugates are not the products of gut microbiota degrading quercetin. Only Jaganath et al. (3) investigated the breakdown of quercetin3-O-rutinoside (rutin) by colonic microflora and deduced that its catabolites may play a key role in the overall antioxidant capacity of the colonic lumen after the ingestion of quercetin-rich foods. This paper showed that the metabolites of all strains had a lower DPPH and $\mathrm{OH}$ free radicals scavenging ability than that of quercetin at almost all doses of quercetin. But the MSCs of $C$. perfringens and B. fragilis showed an approximate activity with about $1 \mathrm{mg} / \mathrm{mL}$ quercetin added in broth. However, $C$. perfringens and B. fragilis are not the major bacteria in the human gut $(21-23)$. Therefore, the most antioxidant activity of quercetin will lose a lot when it reaches the human colon.

Even though the metabolites of seven gut bacteria fermenting quercetin were not fully detected (11), this will not affect the significance of the results presented about antioxidant activities of the fermented broths by seven strains since the mixed system (fermented broth) was complicated and similar to the contents of the human colon in some ways.

It is the first study to report that $C$. perfringens and B. fragilis can degrade quercetin into the same metabolite, 3,4-dihydroxyphenylacetic acid, and that antioxidant activities declined when quercetin was fermented by seven gut bacteria.

\section{Acknowledgements}

We thank Zhen Zhang from the Department of Food Science and Engineering and technicians from Analytical and Testing Center, Jinan University for their contribution to the experiments of this study.

\section{Conflict of interest and funding}

The program was supported by the funds of the National Natural Science Funds (No. 31271849), the Open Project Program of State Key Laboratory of Food Science and Technology of Nanchang University (No. SKLFKF-201212), National Innovation Experiment Program for University Students (No. 101055902), the Fundamental Research Funds for the Central Universities (No. 21611414).

\section{References}

1. Havsteen BH. The biochemistry and medical significance of the flavonoids. Pharmacol Ther 2002; 96: 167-202.
2. Crozier A, Jaganath IB, Clifford MN. Dietary phenolics: chemistry, bioavailability and effects on health. Nat Prod Rep 2009; 26: 1001-43.

3. Jaganath B, Mullen W, Lean MEJ, Edwards CA, Crozier A. In vitro catabolism of rutin by human fecal bacteria and the antioxidant capacity of its catabolites. Free Radic Biol Med 2009; 47: 1180-9.

4. Spencer JPE. Metabolism of tea flavonoids in the gastrointestinal tract. J Nutr 2003; 133: 3255-61.

5. Halliwell B, Rafter J, Jenner A. Health promotion by flavonoids, tocopherols, tocotrienols, and other phenols: direct or indirect effects? Antioxidants or not? Am J Clin Nutr 2005; 81: $268-76$.

6. Gordon MH, Roedig-Penman A. Antioxidant activity of quercetinand myricetin in liposomes. Chem Phys Lipids 1988; 97: $79-85$.

7. Ameho CK, Chen CYO, Smith D, Sánchez-Moreno C, Milbury PE, Blumberg JB. Antioxidant activity and metabolite profile of quercetin in vitamin-E-depleted rats. J Nutr Biochem 2008; 19: $467-74$.

8. Lotito SB, Zhang W, Yang CS, Crozier A, Frei B. Metabolic conversion of dietary flavonoids alters their anti-inflammatory and antioxidant properties. Free Radical Biol Med 2011; 51: 454-63.

9. Serra A, Macia A, Romero M, Reguant J, Ortega N, Motilva M. Metabolic pathways of the colonic metabolism of flavonoids (flavonols, flavones and flavanones) and phenolic acids. Food Chem 2012; 130: 383-93.

10. Yang J, Qian D, Guo J, Jiang S, Shang E, Duan J, $\mathrm{Xu}$ Jun. Identification of the major metabolites of hyperoside produced by the human intestinal bacteria using the ultra performance liquid chromatography/quadrupole-timeof-flight mass spectrometry. J Ethnopharmacol 2013; 147: 174-9.

11. Zhang Z, Peng X, Li S, Zhang N, Wang Y, Wei H. Isolation and identification of quercetin degrading bacteria from human fecal microbes. PLOS one 2014; 9: 1-4.

12. Schneider H, Simmering R, Hartmann L, Pforte H, Blaut M. Degradation of quercetin-3-glucoside in gnotobiotic rats associated with human intestinal bacteria. J Appl Microbiol 2000; 89: $1027-37$.

13. Keppler K, Hein EM, Humpf HU. Metabolism of quercetin and rutin by the pig caecal microflora prepared by freezepreservation. Mol Nutr Food Res 2006; 50: 686-95.

14. Blois MS. Antioxidants determination by the use of a stable free radical. Nature 1958; 181: 1199-200.

15. Halliwell B, Gutteridge JM, Aruoma OI. The deoxyribose method: a simple "test tube" assay for determination of rate constants for reactions of hydroxyl radicals. Anal Biochem 1987; 165: 215-19.

16. Walle TU, Walle K, Halushka PV. Carbon dioxide is the major metabolite of quercetin in humans. J Nutr 2001; 131: $2648-52$.

17. Shirai M, Moon J, Tsushida T, Terao J. Inhibitory effect of a quercetin metabolite, quercetin 3-O- $\beta$-D-glucuronide, on lipid peroxidation in liposomal membranes. J Agric Food Chem 2001; 49: 5602-8.

18. Angeloni C, Spencer JPE, Leoncini E, Biagi PL, Hrelia S. Role of quercetin and its in vivo metabolites in protecting $\mathrm{H} 9 \mathrm{c} 2$ cells against oxidative stress. Biochimie 2007; 89: $73-82$.

19. Chao C, Hou Y, Chao PL, Weng C, Ho F. The antioxidant effects of quercetin metabolites on the prevention of high 
glucose-induced apoptosis of human umbilical vein endothelial cells. Br J Nutr 2009; 101: 1165-70.

20. Dueñas M, González-Manzano S, González-Paramás A, Santos-Buelga C. Antioxidant evaluation of O-methylated metabolites of catechin, epicatechin and quercetin. J Pharm Biomed Anal 2010; 51: 443-9.

21. Wexler HM. Bacteroides: the good, the bad, and the nitty-gritty. Clin Microbiol Rev 2007; 20: 593-621.

22. Tannock GW. The bowel microbiota and inflammatory bowel diseases. Int J Inflamm 2011; 34: 147-54.

23. Tannock GW, Lawley B, Munro Lay KC, Taylor C, Daynes C, Baladjay L, et al. Comprehensive analysis of the bacterial content of stool from patients with chronic pouchitis, normal pouches, or familialadenomatous polyposis pouches. Inflamm Bowel Dis 2012; 18: 925-34.

\section{*Hua Wei}

State Key Laboratory of Food Science and Technology Nanchang University

No. 235, Nanjing Road East

Nanchang City, Jiangxi Province

China

Email: weihual |4@live.cn

*Xichun Peng

Department of Food Science and Engineering

Jinan University

No. 60I, Haungpu Road West

Tianhe Discrict, Guangzhou City, Guang Dong Province

China

Email: tpxchun@jnu.edu.cn 